\title{
Small hairpin RNA-mediated Krüppel-like factor 8 gene knockdown inhibits invasion of nasopharyngeal carcinoma
}

\author{
JING WANG ${ }^{1}$, JIAN LI HU ${ }^{1}$, RU BO CAO ${ }^{1}$, QIAN DING ${ }^{1}$, GANG PENG $^{1}$, SHI JIANG FEI ${ }^{1}$, YAO JIANG ${ }^{1}$, \\ PENG CHENG LI ${ }^{1}$, KUN YU YANG ${ }^{1}$, WEN JIE ZHANG ${ }^{2}$, GANG WU ${ }^{1 *}$, \\ RUO ZHENG WANG ${ }^{3 *}$ and PIN DONG $\mathrm{LI}^{1 *}$ \\ ${ }^{1}$ Cancer Center, Union Hospital, Tongji Medical College, Huazhong University of Science and Technology, Wuhan, \\ Hubei 430023; ${ }^{2}$ Department of Pathology, Shihezi University School of Medicine, Shihezi, Xinjiang 832002; \\ ${ }^{3}$ Department of Radiation Oncology, Affiliated Tumor Hospital, Xinjiang Medical University, \\ Urumqi, Xinjiang 830011, P.R. China
}

Received May 31, 2014; Accepted January 29, 2015

DOI: $10.3892 / \mathrm{ol} .2015 .3099$

\begin{abstract}
The present study aimed to characterize the expression of Krüppel-like factor 8 (KLF8) in nasopahryngeal carcinoma (NPC) cell lines and determine its effect on tumor development and invasion following KLF8 gene knockdown by small hairpin RNA (shRNA). KLF8 expression in four NPC cell lines was examined by quantitative polymerase chain reaction (qPCR) and western blotting. KLF8 was knocked down in the SUNE1-5-8F/Sh-KLF8 cell line using shRNA, and the resulting stable cell line SUNE1-5-8F-sh-KLF8 was transplanted into nude mice in order to observe tumor formation and invasion. The results obtained from qPCR and western blotting revealed that, of the four NPC cell lines, KLF8 expression was lowest in the CNE-1 cells and highest in the SUNE1-5-8F cells. The tumor xenograft mouse models revealed that SUNE1-5-8F/Sh-KLF8 cells had a reduced ability for tumor formation and invasion compared with the control group. These results demonstrated for the first time that KLF8 modulates the formation and invasive ability of nasopharyngeal carcinoma.
\end{abstract}

Correspondence to: Professor Pin Dong Li, Cancer Center, Union Hospital, Tongji Medical College, Huazhong University of Science and Technology, 156 Xinghua Road West, Wuhan, Hubei 430023, P.R. China

E-mail: 1pd8204@163.com

Professor Ruo Zheng Wang, Department of Radiation Oncology, Affiliated Tumor Hospital, Xinjiang Medical University, 789 Shuzhou Road East, Urumqi, Xinjiang 830011, P.R. China E-mail:wrz8526@163.com

*Contributed equally

Key words: Krüppel-like factor 8, nasopharyngeal carcinoma, tumor promoter, expression, invasion

\section{Introduction}

Nasopharyngeal carcinoma (NPC) is a tumor arising from the epithelial cells covering the nasopharynx surface. The highest incidence of NPC worldwide has been reported in Southern China, with an age-standardized incidence rate of 20-50 cases per 100,000 people (1). Viral infections, genetic alterations and environmental factors are considered to contribute towards the initiation and development of NPC (2-5). Currently, radiation therapy is the primary treatment strategy for NPC (6). However, despite improvements in radiotherapy equipment and techniques, the five-year survival rate of NPC patients remains at 50-60\%. Therefore, understanding the key genes involved in the development of NPC is required to identify new therapeutic targets for the treatment of this disease (7).

Krüppel-like factor 8 (KLF8) is a member of the KLF family of proteins, which are essential in cell cycle progression, epithelial-mesenchymal transition (EMT), invasion, oncogenic transformation and cancer development (8-10). Several studies have reported KLF8 overexpression in breast cancer, hepatocellular carcinoma and renal cancer (11-13); therefore, KLF8 may function as a predictor of metastasis and overall survival in certain types of cancer. However, the expression of KLF8 in NPC cell lines and its effects on NPC cells have not yet been investigated.

The present study aimed to evaluate the role of KLF8 in the pathogenesis of NPC by assessing its expression in NPC cell lines. Small hairpin RNA (shRNA) was used to knock down KLF8 in the NPC cell line, SUNE1-5-8F, which was subsequently transplanted into severe combined immunodeficiency (SCID) mice to investigate the tumor formation and invasion.

\section{Materials and methods}

Cell culture and sample collection. The NPC cell lines, CNE1, CNE2, CEN1-LMP1 and SUNE1-5-8F, were obtained from the Cancer Research Institute of Southern Medical University (Guangzhou, China). All the cell lines were maintained in 
RPMI 1640 medium, supplemented with $10 \%$ newborn calf serum (PAA Laboratories, Inc., Pasching, Austria) and incubated in a humidified atmosphere of $5 \% \mathrm{CO}_{2}$ at $37^{\circ} \mathrm{C}$.

Extraction of RNA and quantitative polymerase chain reaction $(q P C R)$. Total RNA was extracted using TRIzol reagent (Invitrogen Life Technologies, Carlsbad, CA, USA), according to the manufacturer's instructions. Subsequently, RNA $(2 \mu \mathrm{g})$ was reverse-transcribed into first-strand cDNA using M-MLV Reverse Transcriptase (Promega Corporation, Madison, WI, USA) according to the manufacturer's instructions. KLF8 and GAPDH were amplified by qPCR using the following primers: KLF8 forward, 5'-GGGTGTTTGGCTTCTTTGC-3', and reverse, 5'-GGCTGTGGTCTCATCTGC-3'; and GAPDH forward, 5'-CTCCTCCTGTTCGACAGTCAGC-3', and reverse, 5'-CCCAATACGACCAAATCCGTT-3'. Gene-specific amplification was performed using an ABI 7900HT real-time PCR system (Applied Biosystems Life Technologies, Foster City, CA, USA) with a $15 \mu \mathrm{l}$ PCR mix containing $0.5 \mu \mathrm{l}$ cDNA, $7.5 \mu 12 \mathrm{X} \mathrm{SYBR}{ }^{\circledR}$ Green master mix (Invitrogen Life Technologies) and $200 \mathrm{nM}$ of the appropriate primers. Next, the mixture was preheated at $95^{\circ} \mathrm{C}$ for $10 \mathrm{~min}$ and then amplified in 45 cycles of $95^{\circ} \mathrm{C}$ for $30 \mathrm{sec}$ and $60^{\circ} \mathrm{C}$ for $1 \mathrm{~min}$. The resolution curve was measured at $95^{\circ} \mathrm{C}$ for $15 \mathrm{sec}, 60^{\circ} \mathrm{C}$ for $15 \mathrm{sec}$ and $95^{\circ} \mathrm{C}$ for $15 \mathrm{sec}$. Subsequently, the threshold cycle $(\mathrm{Ct})$ value of each sample was calculated, and the relative expression of KLF8 mRNA was normalized against the GAPDH value using the $2^{-\Delta \mathrm{Ct}}$ method.

Western blot analysis. Homogenized tissues were lysed in radioimmunoprecipitation assay lysis buffer, and the lysates were harvested by centrifugation $(8,000 \mathrm{xg})$ at $4^{\circ} \mathrm{C}$ for $30 \mathrm{~min}$. Next, $8 \mu \mathrm{g}$ protein samples were separated by electrophoresis on a $15 \%$ sodium dodecyl sulfate-polyacrylamide gel and were transferred onto a polyvinylidene fluoride membrane. Subsequently, nonspecific binding sites were blocked by placing the membrane in $5 \%$ nonfat milk for $1 \mathrm{~h}$, followed by incubation with a polyclonal rabbit anti-human KLF8 antibody (dilution, 1:1,000; cat. no. 252109; Abbiotec, San Diego, CA, USA) at $4^{\circ} \mathrm{C}$ overnight. After washing four times in Tris-buffered saline with Tween-20, the membrane was probed with a horseradish peroxidase (HRP)-conjugated rabbit anti-rat immunoglobulin $\mathrm{G}$ antibody (dilution, 1:5,000; cat. no. KC-5G5, Proteintech Group, Inc., Chicago, IL, USA) at $37^{\circ} \mathrm{C}$ for $60 \mathrm{~min}$. Next, the samples were washed four times and the bands were detected using Pierce ECL Western Blotting Substrate (Thermo Fisher Scientific, Waltham, MA, USA). Band density was measured using ImageJ software [version 1.43b; National Institutes of Health (NIH), Bethesda, MA, USA] and was standardized against mouse anti-human GAPDH monoclonal antibody (Shanghai Kangcheng Biotechnology Co., Ltd., Shanghai, China).

Lentiviral vector construction and transfection. In order to knockdown the expression of human KLF8, a KLF8-RNA interference lentiviral vector [pGCSIL-green fluorescent protein (GFP)-KLF8 shRNA] was constructed by annealing the KLF8-shRNA (5'-CCGGCTAGCATGCTACAAGCTCCAATT CAAGAGATTGGAGCTTGTAGCATGCTAGTTTTTG-3') and inserting it into the shRNA expression vector,pGCSIL-GFP. A scrambled shRNA was used as a negative control (12). The recombinant virus was packed using the Lentivector Expression system (Shanghai GeneChem Co., Ltd., Shanghai, China) and termed as shRNA-KLF8 lentivirus or negative shRNA lentivirus. Subsequently, SUNE1-5-8F cells were transfected with the recombinant lentivirus using Lipofectamine ${ }^{\circledR} 2000$ (Invitrogen Life Technologies). The transfected cells were screened under $800 \mu \mathrm{g} / \mathrm{ml} \mathrm{G} 418$ (Calbiochen, Darmstadt, Germany) for four weeks to generate two stable monoclonal cell lines (including a KLF8 stable downregulated cell line,SUNE1-5-8F/Sh-KLF8, and a control stable cell line, SUNE1-5-8F/Mock). Western blot analysis was used to determine the expression levels of KLF8 in these cell lines.

Cell invasion assays. A cell invasion assay was performed using a precoated Cell Invasion Assay kit (Chemicon International, Inc., Temecula, CA, USA), according to the manufacturer's instructions. Cells transported from the extracellular matrix layer to the lower surface of the membrane were fixed with methanol and stained with crystal violet (Guangzhou Chemical Reagent Factory, Guangzhou, China). Images of three randomly selected fields of the fixed cells were captured and the cells were counted using an inverted microscope (XDS-1; Olympus Corporation, Tokyo, Japan). The experiments were conducted in triplicate.

In vivo assay for the determination of tumor growth. All the animal experiments were approved by the Ethics Committee on Animal Experimentation of the Huazhong University of Science and Technology (Wuhan, China), and the procedures complied with the NIH Guide for the Care and Use of Laboratory Animals (8th edition, 2011). $\mathrm{BALB} / \mathrm{c}$ nude mice $(\mathrm{Nu} / \mathrm{Nu}$; female; age, 4-6 weeks) were purchased from the Center of Experimental Animal of Huazhong University of Science and Technology and maintained under pathogen-free conditions ( $\mathrm{n}=11$ per group). SUNE1-5-8F/Mock and SUNE1-5-8F/Sh-KLF8 cells were injected into the left and right flanks, respectively, of the mice. Tumor growth was monitored by measuring tumor volume, which was calculated using the following formula: Tumor volume $\left(\mathrm{mm}^{3}\right)=$ width $^{2} \mathrm{x}$ length $(\mathrm{mm}) / 2$. At the end of the experiment, the tumor was harvested. Differences in tumor growth were analyzed for statistical significance.

Statistical analysis. All the experimental data are presented as the mean \pm standard deviation. The mean values of different groups were compared using one-way analysis of variance or Student's t-test. A two-sided $\mathrm{P}<0.05$ was considered to indicate a statistically significant difference. All statistical analyses were performed with the SPSS software (version 16.0; SPSS, Inc., Chicago, IL, USA).

\section{Results}

mRNA and protein KLF8 expression levels in four NPC cell lines. The relative transcriptional level of KLF8 was determined by qPCR and western blot analysis in the four NPC cell lines. KLF8 expression was found to be lowest in the CNE-1 cell line and highest in the SUNE1-5-8F cell line. The mRNA expression levels of KLF8 were consistent with the protein expression levels in all the cell lines (Figs. 1, 2A and 2B). 
Expression of KLF8 in the SUNE1-5-8F cell line is significantly downregulated by the $P G C S I L-G F P-K L F 8$ lentiviral vector. pGCSIL-GFP-KLF8 shRNA was transfected into the SUNE1-5-8F cells in order to knockdown the expression of KLF8 in the cell line. All the untransfected NPC cells were nonviable following G418 (800 $\mu \mathrm{g} / \mathrm{ml})$ selection for one week. Cells transfected with pGCSIL-GFP-KLF8 were continuously selected using G418 for four weeks until clones were observed using fluorescence microscopy.

The knockdown efficiency in SUNE1-5-8F cells was examined by western blot analysis of KLF8 protein expression. KLF8 expression was significantly reduced in cells transfected with pGCSIL-GFP-KLF8 compared with the SUNE1-5-8F and SUNE1-5-8F/Mock cells ( $\mathrm{P}<0.05$; Fig. 3).

KLF8 knockdown reduces cell invasion ability. The transwell invasion assay revealed that knockdown of KLF8 resulted in a reduction of the invasiveness of NPC cells, as indicated by a significant decrease in the number of invaded cells $(\mathrm{P}=0.0005$; Fig. 4).

Downregulation of KLF8 expression suppresses tumor growth in vivo. The effect of reduced KLF8 expression on tumor growth was investigated in NPC xenografts. Two experimental groups were examined: i) SUNE1-5-8F/Mock cells; and ii) SUNE1$5-8 \mathrm{~F} / \mathrm{Sh}-\mathrm{KLF} 8$ cells. Tumor growth curves were plotted to compare the difference in tumor formation throughout the experiment. Palpable tumors were initially detected in all the mice at day 11 following injection. The tumor volume differed significantly at day 23 (Fig. 5A). The mean tumor volume produced by injection with SUNE1-5-8F/Sh-KLF8 was $>50 \%$ lower $(\mathrm{P}<0.05$; Fig. 5B) compared with the control group. The mean tumor weight was also significantly reduced in SUNE1-5-8F/Sh-KLF8 tumors ( $\mathrm{P}<0.05$; Fig. 5B).

\section{Discussion}

NPC is a serious healthcare problem (14), more frequently occurring in Southern China, which presents early metastasis features. At present, radiation therapy is the main NPC treatment. The five-year survival rate remains at $50-60 \%$, in spite of advances in radiotherapy devices and techniques. Therefore, comprehensive research into new therapies for the treatment of NPC are required. A greater understanding of the key molecules involved in tumor development may provide new therapeutic targets to develop more effective treatments for the NPC.

KLF8 is a GT-box (CACCC) binding dual-transcription factor, which is widely expressed in adult tissues, particularly in the kidney, heart and placenta. KLF8 plays an important role in the regulation of cell cycle progression (8), transformation (15), EMT and invasion (10) in various types of cancer. However, the expression of KLF8 and its role in NPC cells have not been previously investigated.

To the best of our knowledge, the present study is the first to characterize the expression of KLF8 in NPC cell lines and determine the tumor formation and invasion ability in these cells following KLF8 gene knockdown by shRNA. The mRNA and protein expression levels of KLF8 in four NPC cell lines were assessed by qPCR and western blot analysis, revealing that KLF8 expression was lowest in the CNE-1 cells

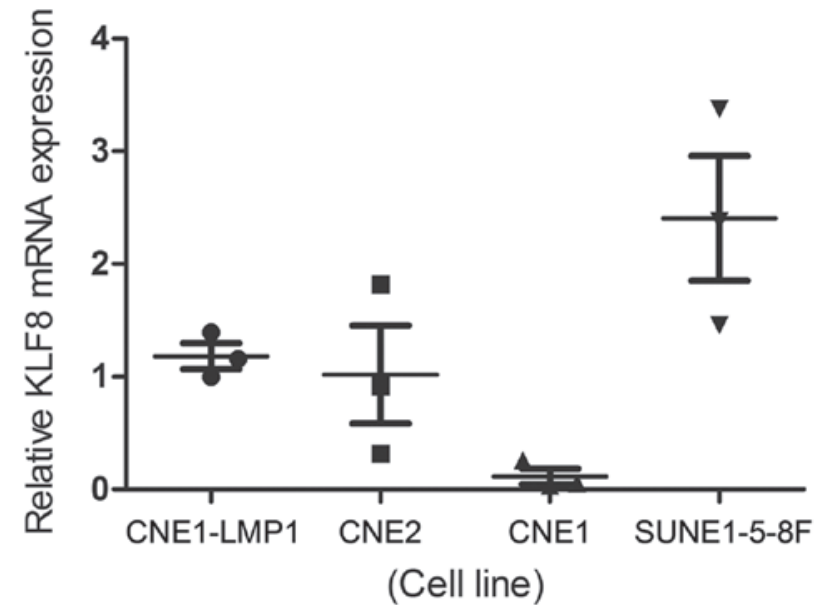

Figure 1. KLF8 mRNA expression in human nasopharyngeal carcinoma cell lines, CNE1, CNE2, CEN1-LMP1 and SUNE1-5-8F, assessed by quantitative polymerase chain reaction. KLF8 expression was lowest in the CNE-1 cell line and highest in the SUNE1-5-8F cell line. KLF8, Krüppel-like factor 8.

A

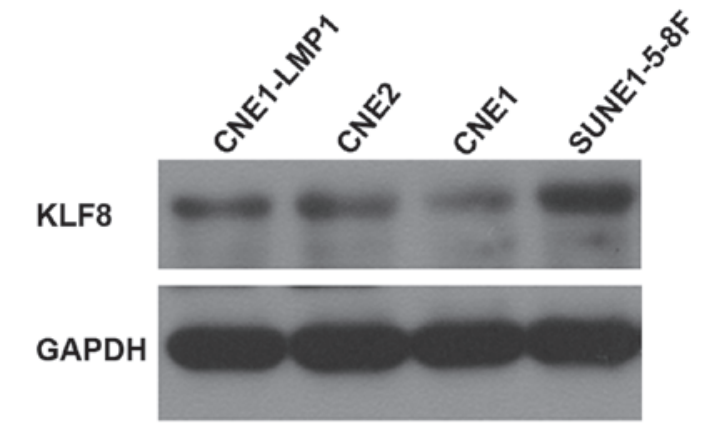

B

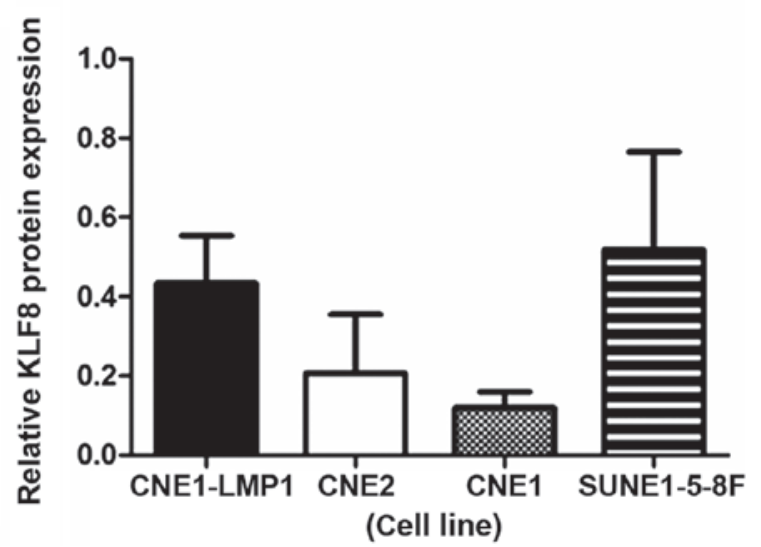

Figure 2. (A) KLF8 protein expression in human NPC cell lines, CNE1, CNE2, CEN1-LMP1 and SUNE1-5-8F, assessed by western blotting. (B) The protein expression levels in the four NPC cell lines were consistent with the mRNA expression of KLF8 in the four NPC cell lines. KLF8, Krüppel-like factor 8; NPC, nasopharyngeal carcinoma.

and highest in the SUNE1-5-8F cells. The mRNA expression of KLF8 was consistent with its protein expression in the four cell lines. Notably, the expression of KLF8 was consistent with the malignancy degree of the NPC cell lines, which indicated that KLF8 played an important role in the transformation from normal phenotype to malignant phenotype. A study by Fu et al (12) demonstrated that the expression of KLF8 protein and mRNA is higher in tumorous tissue compared with 
A

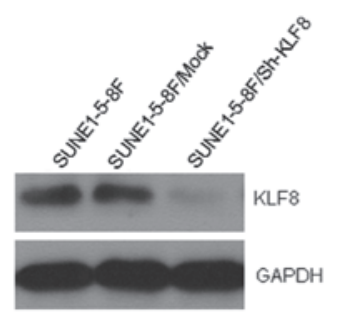

B

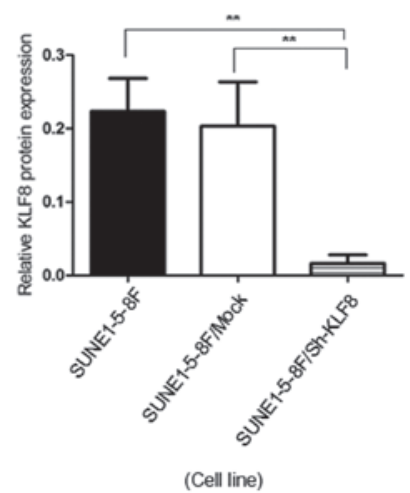

Figure 3. Effect of KLF8-targeting shRNAs on KLF8 expression. (A) Immunoblot analysis of KLF8 in SUNE1-5-8F cells indicated downregulation of KLF8 protein in shRNA. (B) The result demonstrated that the protein levels of KLF8 in pGCSIL-green fluorescent protein-KLF8 cells were significantly weaker compared with the protein levels in the SUNE1-5-8F and SUNE1-5-8F/Mock cells ${ }^{* *} \mathrm{P}<0.01$, SUNE1-5-8F/Sh-KLF8vs.SUNE1-5-8F,SUNE1-5-8F/ Sh-KLF8 vs. SUNE1-5-8F/Mock). KLF8, Krüppel-like factor 8; shRNA, small hairpin RNA.
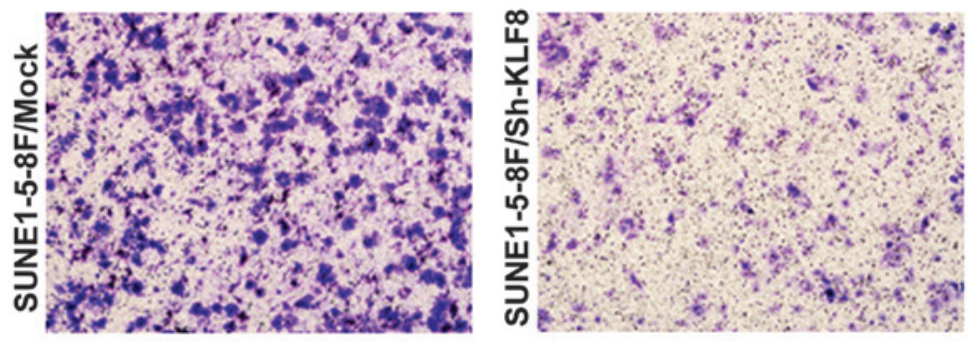

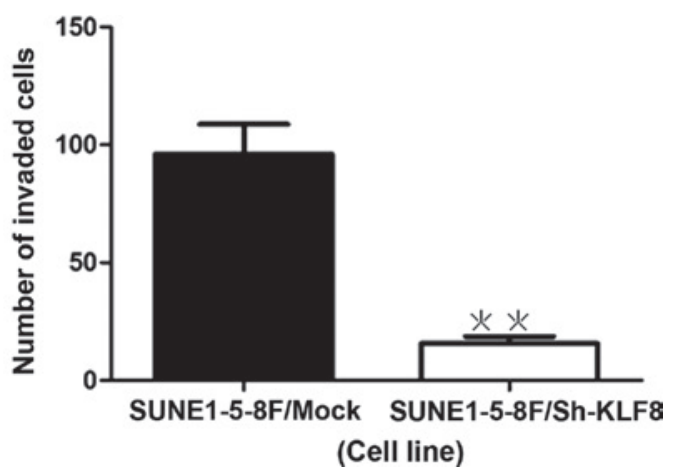

(Cell line)

Figure 4. Comparison of in vitro invasion potency of SUNE1-5-8F/Mock and SUNE1-5-8F/Sh-KLF8 cells using a cell invasion assay. Each bar represents the mean \pm standard deviation of three independent experiments ( ${ }^{* *} \mathrm{P}<0.01 \mathrm{vs.} \mathrm{SUNE1-5-8F/Mock).} \mathrm{The} \mathrm{results} \mathrm{demonstrated} \mathrm{that} \mathrm{knockdown} \mathrm{of} \mathrm{KLF8} \mathrm{substantially}$ reduced the invasiveness of nasopharyngeal carcinoma cells, as indicated by a marked decrease in the number of invaded cells. KLF8, Krüppel-like factor 8 .
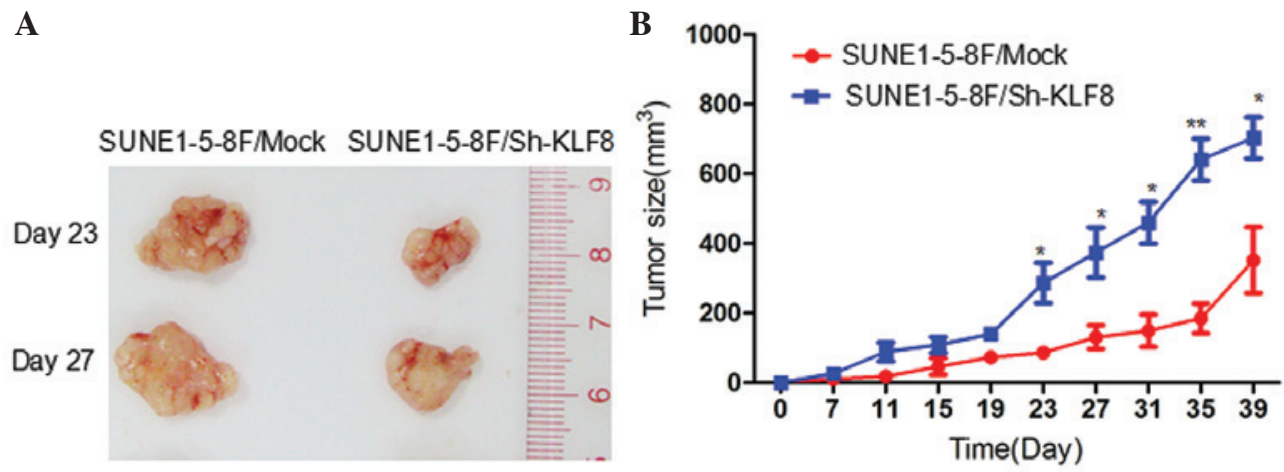

Figure 5. Downregulation of KLF8 expression suppress tumor growth in vivo. (A) KLF8 knockdown nasopharyngeal carcinoma cells were injected subcutaneously into the right flank of nude mice. Control cells were injected subcutaneously into the left flank of the same nude mice ( $\mathrm{n}=11)$. At six weeks following implantation, KLF8 knockdown cells produced smaller tumors compared with the control cells. (B) The tumor from each group was measured immediately following the dissection. The average tumor size is presented as the mean \pm standard deviation ( ${ }^{*} \mathrm{P}<0.05, \mathrm{SUNE} 1-5-8 \mathrm{~F} / \mathrm{Sh}-\mathrm{KLF} 8$ vs. SUNE1-5-8F/Mock on days 23, 27, 31 and 39.and "*P<0.01, SUNE1-5-8F/Sh-KLF8 vs. SUNE1-5-8F/Mock on day 35). KLF8, Krüppel-like factor 8.

normal tissue. Another study (13) revealed that the expression of KLF8 was higher in hepatocellular carcinoma with high metastatic ability. The aforementioned observations indicate that KLF8 promotes tumor development and is important in the processes of invasion and metastasis.

In order to assess the effect of KLF8 on tumor growth and invasion in NPC cells, in the current study, a lentiviral vector was constructed and transfected into the SUNE1-5-8F cell line. The findings revealed that the protein expression levels of KLF8 in pGCSIL-GFP-KLF8-transfected SUNE1-5-8F cells were significantly lower compared with SUNE1-5-8F/ Mock and SUNE1-5-8F/Sh-KLF8 cells.

The effect of KLF8 downregulation on tumor growth was also evaluated using an NPC xenograft, which revealed that the average tumor volume of the SUNE1-5-8F/Sh-KLF group was significantly lower compared with the SUNE1-5-8F/Mock group. 
In conclusion, the results demonstrated that, of four NPC cell lines, KLF8 expression was lowest in the CNE-1 cell line and highest in the SUNE1-5-8F cell line. The mRNA expression levels of KLF8 were consistent with the protein expression levels in the four cell lines. Compared with the control group, downregulation of KLF8 expression is able to reduce the tumor growth in nasopharyngeal carcinoma xenografts. The present study indicated that KLF8 is important in NPC development. Further elucidation of the underlying molecular mechanisms of KLF8 in NPC may allow for the development of a novel molecular targeted therapy for the treatment of this disease.

\section{Acknowledgements}

This study was supported by the National Natural Science Foundation of China (grant no. 81160327, awarded to Ruo Zheng Wang), the Union Hospital Key Laboratory Foundation of Biological Target Therapy (grant no. 02.03.2013-80, awarded to Pin Dong Li) and the Independent Innovation Research Foundation of Huazhong University of Science and Technology (grant no. 01-08-530059, awarded to Pin Dong Li).

\section{References}

1. Yao KT: The application and prospect of nasopharyngeal carcinoma etiology. China Cancer 6: 3-4, 1997 (In Chinese).

2. Fang W, Li X, Jiang Q, et al: Transcriptional patterns, biomarkers and pathways characterizing nasopharyngeal carcinoma of Southern China. J Transl Med 6: 32, 2008.

3. Alajez NM, Shi W, Hui AB, et al: Enhancer of Zeste homolog 2 (EZH2) is overexpressed in recurrent nasopharyngeal carcinoma and is regulated by miR-26a, miR-101, and miR-98 Cell Death Dis 1: e85, 2010.
4. Liu Z, Luo W, Zhou Y, et al: Potential tumor suppressor NESG1 as an unfavorable prognosis factor in nasopharyngeal carcinoma. PLoS One 6: e27887, 2011.

5. Xiong S, Wang Q, Zheng L, Gao F and Li J: Identification of candidate molecular markers of nasopharyngeal carcinoma by tissue microarray and in situ hybridization. Med Oncol 28: S341-S348, 2011.

6. Lee AW, Ng WT, Chan YH, et al: The battle against nasopharyngeal cancer. Radiother Oncol 104: 272-278, 2012.

7. Zhen Y, Ye Y, Yu X, et al: Reduced CTGF expression promotes cell growth, migration, and invasion in nasopharyngeal carcinoma. PLoS One 8: e64976, 2013.

8. Urvalek AM, Wang X, Lu H and Zhao J: KLF8 recruits the p300 and PCAF co-activators to its amino terminal activation domain to activate transcription. Cell Cycle 9: 601-611, 2010.

9. Wang X, Zheng M, Liu G, et al: Krüppel-like factor 8 induces epithelial to mesenchymal transition and epithelial cell invasion. Cancer Res 67: 7184-7193, 2007.

10. Mehta TS, Lu H, Wang X, et al: A unique sequence in the $\mathrm{N}$-terminal regulatory region controls the nuclear localization of KLF8 by cooperating with the C-terminal zinc-fingers. Cell Res 19: 1098-1109, 2009.

11. Wang X, Lu H, Urvalek AM, et al: KLF8 promotes human breast cancer cell invasion and metastasis by transcriptional activation of MMP9. Oncogene 30: 1901-1911, 2011.

12. Fu WJ, Li JC, Wu XY, et al: Small interference RNA targeting Krüppel-like factor 8 inhibits the renal carcinoma 786-0 cells growth in vitro and in vivo. J Cancer Res Clin Oncol 136: 1255-1265, 2010.

13. Li JC, Yang XR, Sun HX, et al: Up-regulation of Krüppel-like factor 8 promotes tumor invasion and indicates poor prognosis for hepatocellular carcinoma. Gastroenterology 139: 2146-2157, 2010.

14. Chen MK, Chen TH, Liu JP, et al: Better prediction of prognosis for patients with nasopharyngeal carcinoma using primary tumor volume. Cancer 100: 2160-2166, 2004.

15. Zhao J, Bian ZC, Yee K, et al: Identification of transcription factor KLF8 as a downstream target of focal adhesion kinase in its regulation of cyclin D1 and cell cycle progression. Mol Cell 11: 1503-1515, 2003. 